\title{
Regulation of mouse embryonic stem cell self-renewal by a Yes-YAP-TEAD2 signaling pathway downstream of LIF
}

\author{
Christoffer Tamm, Nathalie Böwer and Cecilia Annerén* \\ Department of Medical Biochemistry and Microbiology, Uppsala University, 75123 Uppsala, Sweden \\ *Author for correspondence (cecilia.anneren@imbim.uu.se) \\ Accepted 18 November 2010 \\ Journal of Cell Science 124, 1136-1144 \\ (C) 2011. Published by The Company of Biologists Ltd \\ doi:10.1242/jcs.075796
}

\section{Summary}

The cytoplasmic tyrosine kinase Yes has previously been shown to have an important role in maintaining mouse and human embryonic stem (ES) self-renewal through an unknown pathway downstream of leukemia inhibitory factor (LIF) and one or more factors in serum. Here, we show that TEAD2 and its transcriptional co-activator, the Yes-associated protein YAP, co-operate in a signaling pathway downstream of Yes. We show that YAP, TEAD2 and Yes are highly expressed in self-renewing ES cells, are activated by LIF and serum, and are downregulated when cells are induced to differentiate. We also demonstrate that kinase-active Yes binds and phosphorylates YAP, and activates YAP-TEAD2-dependent transcription. We found that TEAD2 associates directly with the Oct-3/4 promoter. Moreover, activation of the Yes pathway induced activity of the Oct-3/4 and Nanog promoters, whereas suppression of this pathway inhibited promoter activity. Nanog, in turn, suppressed TEAD2-dependent promoter activity, whereas siRNA-mediated knockdown of Nanog induced it, suggesting a negative regulatory feedback loop. Episomal supertransfection of cells with inhibitory TEAD2-EnR induced endodermal differentiation, which suggests that this pathway is necessary for ES cell maintenance.

Key words: Embryonic stem cells, Leukemia inhibitory factor, Self-renewal, Src family kinases, Yes-associated protein

\section{Introduction}

Pluripotent embryonic stem (ES) cells can be expanded in culture and induced to differentiate into all three germ layers. Self-renewal of mouse ES cells depends on the LIF-induced activation of the signal transducer gp130 (also known as interleukin 6 signal transducer, IL6ST), and withdrawal of LIF induces differentiation (Burdon et al., 2002). Gp130 activates at least four pathways: the signal transducer and activator of transcription 3 (STAT3) pathway, the phosphoinositide 3-kinase (PI3K) pathway, the mitogenactivated protein kinase (MAPK) pathway and the Src family kinase (SFK) pathway (Annerén, 2008; Burdon et al., 2002; Liu et al., 2007). The STAT3, PI3K and SFK pathways help to sustain self-renewal, whereas activation of the MAPK pathway induces differentiation (Burdon et al., 1999). It was recently shown that the STAT3 pathway activates mainly SOX2, whereas PI3K predominantly activates Nanog (Niwa et al., 2009). However, no downstream target of LIF-activated SFKs has to date been identified in ES cells.

The Src family consists of nine members and has been shown to regulate a wide variety of cellular functions, such as proliferation, differentiation and survival (Abram and Courtneidge, 2000; Thomas and Brugge, 1997). Because all these processes are involved in embryogenesis, it is not surprising that seven SFKs have so far been identified to be expressed in ES cells and at least three of these, Yes, Hck and Lck, undergo dynamic changes in transcriptional and post-transcriptional regulation during human and mouse ES cell differentiation and are activated by LIF (Annerén et al., 2004; Ernst et al., 1994; Meyn et al., 2005). Interestingly, it was recently proposed that individual SFKs control distinct and opposing pathways in ES cell with Yes, Hck and Lck supporting self-renewal, whereas Src, which remains highly expressed in ES cells during differentiation, promotes differentiation into primitive ectoderm (Meyn et al., 2005; Meyn and Smithgall, 2009).

We have focused our attention on Yes because several findings suggest that this SFK has a particularly important role in selfrenewing ES cells: (1) two independent transcriptional profiling screens found that Yes was highly enriched in ES cells compared with differentiated cells (Ivanova et al., 2002; Ramalho-Santos et al., 2002); (2) a transcriptome study specifically identified Yes as a 'Pluri' gene (a LIF-dependent gene, highly expressed in pluripotent cells, whose expression levels decreases sharply upon LIF withdrawal) (Trouillas et al., 2009) and Yes kinase activity is downregulated in response to differentiation in mouse and human ES cells (Annerén et al., 2004); (3) LIF and serum activates Yes in ES cells (Annerén et al., 2004); (4) specific knockdown of Yes with small interfering RNAs leads to mouse ES cell differentiation and reduced expression of key ES cell pluripotency genes including Nanog and Oct-3/4 (also known as POU domain, class 5, transcription factor 1 or Pou5f1) (Annerén et al., 2004); and finally, (5) Oct3/4 has been shown to associate with the Yes promoter and positively regulate Yes expression, suggesting that Yes is a downstream target gene of Oct3/4 (Zhang et al., 2007).

Whilst searching for potential signaling pathways downstream of Yes, we found that the Yes-associated protein YAP (YAP65/YAP1) and a member of the TEA DNA binding domain protein family TEAD2 (TEF4) co-operate in a signaling pathway downstream of Yes. YAP is a powerful transcriptional co-activator, which is heavily involved in many pathways that regulate proliferation and cell death, and there is currently an upsurge in studies examining the role of YAP in various cancers (Zhao et al., 
2008). As the name implies, YAP was first identified as a Yes binding partner (Sudol, 1994), but despite the fact that the prolinerich region of YAP was found to associate with the Src homology domain 3 (SH3) of Yes many years ago (Sudol, 1994), only one study has since demonstrated a specific role of SFKs in YAPmediated signaling (Zaidi et al., 2004). YAP interacts with SFKs at the plasma membrane, with 14-3-3 in the cytoplasm and with transcription factors in the nucleus (Basu et al., 2003; Vassilev et al., 2001; Zaidi et al., 2004). Inhibition of the YAP-related pathway is mediated by serine phosphorylation of YAP by the hippo pathway or Akt, which induces binding of 14-3-3 and retention in the cytoplasm (Basu et al., 2003; Zhao et al., 2007). Levels of Yap mRNA are enriched in mouse ES cells (Ramalho-Santos et al., 2002), but YAP is also present in most mouse and human tissues and cell types ranging from two-cell embryos to adults (Sudol et al., 1995).

Mammals express four highly conserved TEAD proteins that regulate transcription of a variety of genes and serve different functions during development. TEAD proteins share a highly conserved 68 amino acid TEA/ATTS DNA-binding domain, which binds to reporter elements related to the consensus sequence $\mathrm{G}(\mathrm{A}) \mathrm{GA}(\mathrm{T} / \mathrm{C}) \mathrm{ATG}$ in gene promoters or enhancers (Jiang et al., 2000). All TEAD proteins require a transcriptional co-activator to function, of which the predominant, if not exclusive one is YAP, and overexpression of TEAD proteins without YAP results in quenching of transcriptional activity (Jiang and Eberhardt, 1995; Vassilev et al., 2001). TEAD2 and TEAD4 are predominantly expressed during early embryogenesis (Kaneko et al., 1997; Yagi et al., 2007). However, although TEAD4 is primarily expressed in the trophectoderm, TEAD2 is expressed in both inner cell mass (ICM) and trophectoderm of the blastocysts at fairly equal levels (Kaneko et al., 1997; Kaneko et al., 2004; Yagi et al., 2007). Furthermore, TEAD2 is highly enriched in ES cells (RamalhoSantos et al., 2002). However, TEAD2-knockout mice (Tead2 ${ }^{--}$) exhibit no obvious abnormalities and are fertile, which is believed to be due to functional redundancy with TEAD1 (Sawada et al., 2008). Additionally, although Tead $2^{--} ;$Tead $^{-/}$double mutants are morphologically identical to wild-type embryos up to day E6.5, they do, however, exhibit growth retardation and severe morphological abnormalities by E8.5 and have a phenotype that is similar to YAP mutant mice (Morin-Kensicki et al., 2006; Sawada et al., 2008). A role of YAP and TEAD4 for specification of trophectoderm in pre-implantation mouse embryos was recently established, and it was demonstrated that expression of constitutively active TEAD4 (TEAD4-vp16) is sufficient to promote trophoblast fate in ES cells (Nishioka et al., 2009; Nishioka et al., 2008; Yagi et al., 2007).

In the present study, we examine the role of Yes, YAP and TEAD2 in ES cells. We show that TEAD2 activity is necessary for ES cell self-renewal and that Yes, YAP and TEAD2 are parts of the same signaling pathway, downstream of LIF, that induces expression of Oct3/4.

\section{Results}

Dynamic changes in transcriptional and post-translational regulation of Yes, YAP and TEAD2 during mouse ES cell differentiation

Real-time quantitative PCR (qPCR) data confirmed that Tead2 is the pre-dominantly expressed TEAD member in mouse ES cells, whereas small or near undetectable levels of Teadl, Tead 3 and Tead 4 mRNA were found (Fig. 1A). However, when ES cells were
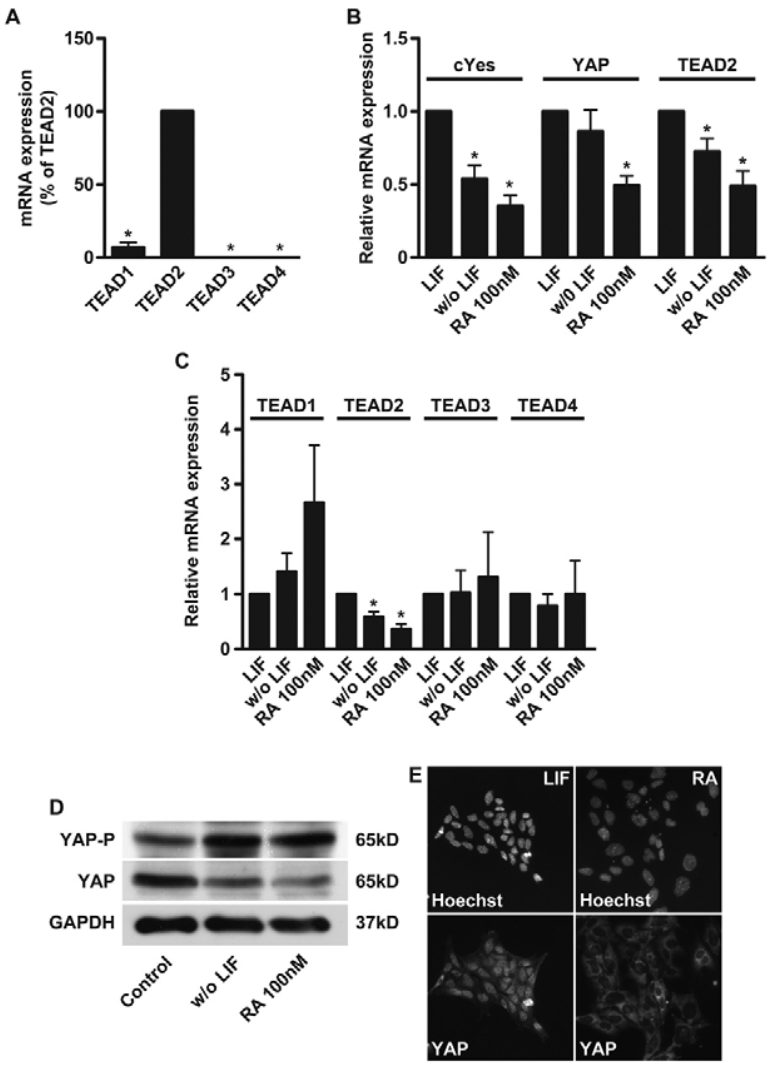

Fig. 1. Yes, YAP and TEAD2 are highly expressed in undifferentiated ES cells and are downregulated in response to differentiation. (A) QPCR analysis of Tead1, Tead2, Tead 3 and Tead4 mRNA expression in E14/T cells. Transcript level comparison was based on primer efficiency estimated from five-point dilution curves and used for comparative $C_{t}$ computation according to the PFAFFL method (Pfaffl, 2001). (B) QPCR analysis of Yes, Yap and Tead 2 mRNA expression in control E14/T cells cultured in the presence of LIF or induced to differentiate in the absence of LIF (w/o LIF) or with $100 \mathrm{nM}$ retinoic acid (RA) for 3 days. Expression of $\beta$-actin is used for normalization, and the calibrator controls were cells grown in the presence of LIF. (C) qPCR analysis of the same cDNA samples as in B showing relative Tead1, Tead2, Tead 3 and Tead 4 mRNA expression in response to differentiation. Note that the mRNA expression levels are shown as percentage of internal control (i.e. expression of each gene in undifferentiated ES cells) and that the absolute mRNA levels of Tead1, Tead 3 and Tead 4 are negligible compared with the level of Tead2 (as shown in A). (D) Western blot analysis of total YAP and serine-phosphorylated (Ser127) YAP (YAP-P) expression in cells shown in B. (E) Representative micrographs of undifferentiated E14/T cells (LIF, left panels) and differentiated ES cells exposed to $100 \mathrm{nM}$ retinoic acid for three days (RA, right panels) stained with Hoechst 33342 (top panels) and anti-YAP (bottom panels) antibodies. All qPCR results are mean \pm s.e.m. comparative $C_{t}$ values $(n=3) ;{ }^{*} P<0.05$ (ANOVA; Tukey's MC Test).

induced to differentiate for 3 days, a significant reduction in levels of Tead2, Yap and Yes mRNA were observed (Fig. 1B). The level of Teadl mRNA was slightly, although not significantly, increased in response to differentiation, albeit from a very low basal level, whereas transcription of Tead 3 and Tead4, with expression levels of less than $0.1 \%$ of Tead 2 expression was not increased in response to differentiation (Fig. 1C). Cells, induced to differentiate for 3 days, expressed lower total YAP protein levels but higher levels of serine-phosphorylated YAP compared with levels in undifferentiated ES cells (Fig. 1D). In addition, YAP was predominantly found in 
the nuclei of undifferentiated ES cells, whereas retinoic-acidinduced (100 nM, 3 days) differentiated cells exhibited increased cytosolic YAP, as well as a clear decrease in nuclear YAP localization (Fig. 1E).

Reporter gene constructs expressing the firefly luciferase gene driven by the human chorionic somatomammotropin (CS) promoter with or without a TEAD enhancer region consisting of multiple (24) copies of the TEAD-binding GTIIC (GGAATG) site (pGTIICLuc and pCS-Luc, respectively) (Jiang and Eberhardt, 1995) were expressed in self-renewing or differentiating ES cells and the luciferase activities were measured. Barely detectable luciferase activity was found in cells transfected with pCS-Luc compared with pGTIIC-Luc, demonstrating that the CS promoter in the pGTIIC construct is mainly driven by the TEAD2 enhanson when expressed in ES cells (Fig. 2A). Moreover, TEAD-dependent transcription in ES cells decreased when cells were induced to differentiate for 3 days by withdrawal of LIF or by addition of 100 $\mathrm{nM}$ retinoic acid (RA) (Fig. 2B).

\section{LIF induces TEAD2-dependent transcription in a Yes- and YAP-dependent manner}

To determine whether LIF induces TEAD2-dependent transcription, ES cells transfected with pGTIIC-Luc with or without short hairpin RNA (shRNA) targeting TEAD2 were starved of serum and LIF overnight and then treated for 6 hours with LIF. The results show that LIF activates TEAD2, in agreement with previously described data showing LIF-induced Yes kinase activity (Annerén et al., 2004). shRNA targeting TEAD2 partly inhibited this effect (Fig. $2 \mathrm{C})$. To exclude the possibility that our shRNA constructs have an effect upstream of the LIF receptor, cells were transfected with shRNA targeting YAP, TEAD2 or EGFP and then analyzed for tyrosine-phosphorylated Stat3 (Tyr705) and total Stat3 by western blotting. shRNA against YAP or TEAD2 showed no effect on phosphorylation of Stat3 (supplementary material Fig. S1). Next, the pGTIIC-Luc construct was co-expressed with YAP, Yes, a constitutively kinase active Y535F mutant of Yes (CA-Yes) or vectors expressing shRNA targeting Yes, YAP or TEAD2. Western blot analysis showed that ectopic expression of both Yes and CAYes dramatically increased the level of autophosphorylated (active) Yes in ES cells (supplementary material Fig. S2C). All shRNAs mediated a reduction of the respective mRNA and protein levels in transiently transfected cells as determined by qPCR and western blotting (supplementary material Fig. S2A,B). The endogenous TEAD-dependent transcriptional activity was increased by $50 \%$ in YAP-transfected cells, demonstrating that YAP is a rate-limiting factor for TEAD2-enhanced transcription in ES cells (Fig. 2D). In addition, overexpression of Yes or CA-Yes induced a two- to threefold increase in TEAD2-dependent transcription compared with that in cells transfected with pGTIIC alone, whereas expression of shTEAD2, shYAP or shYes decreased TEAD2 activity (Fig. 2D and supplementary material Fig. S3). Both shTEAD2 and shYAP significantly impaired CA-Yes-induced TEAD2-dependent transcription (Fig. 2E) and concurrently, pre-treatment of cells with the Src family tyrosine kinase inhibitor PP2 $(10 \mu \mathrm{M})$ also markedly reduced the effect of LIF and CA-Yes (data not shown). Together, these results show that, similarly to YAP, Yes induces TEAD-dependent transcription and suggest that Yes is part of a signaling pathway upstream of YAP and TEAD2 in ES cells.

To determine whether LIF and/or SFKs also activate TEAD DNA binding activity, LIF- and serum-starved ES cells were treated with LIF and/or $5 \mu \mathrm{M}$ of the selective SFK inhibitor SU6656 for
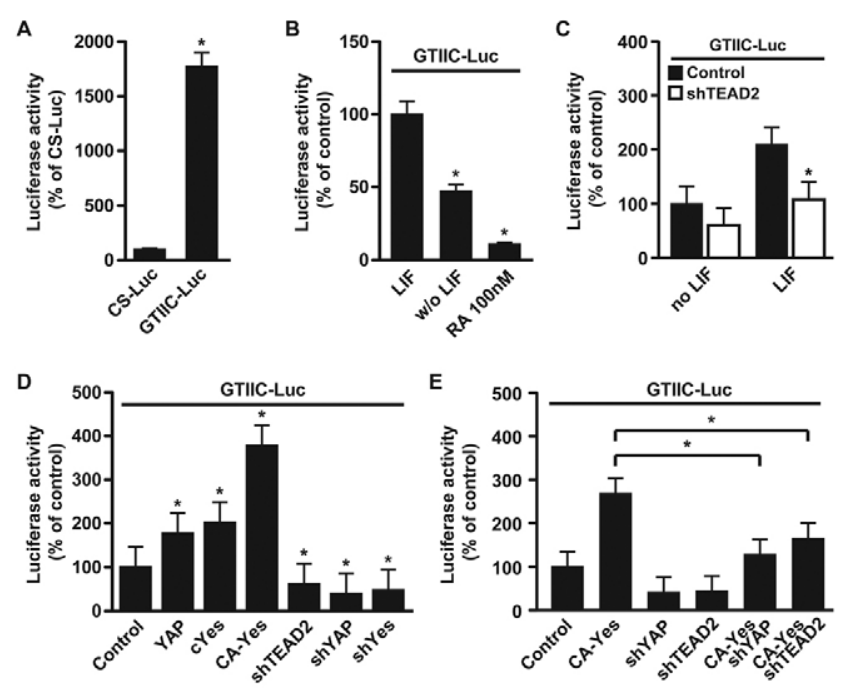

Fig. 2. TEAD2 activity is induced by LIF, YAP and Yes in ES cells. (A) Luciferase activity in E14/T cells transfected with constructs expressing the firefly luciferase gene driven by the human chorionic somatomammotropin (CS) promoter with (pGTIIC-Luc) or without (pCS-Luc) a TEAD2 enhancer region. (B) TEAD2-dependent promoter activity (pGTIIC) in E14/T control cells (LIF) and LIF-starved (w/o LIF) or RA-treated (RA $100 \mathrm{nM}$ ) for 3 days. (C) TEAD2 activity in E14/T cells starved of LIF and serum overnight and treated with LIF with or without the presence of shRNA against TEAD2. (D) TEAD2 activity in E14/T cells transfected with constructs expressing YAP, Yes, CA-Yes or shRNA against YAP, Yes and TEAD2 (analyzed 48 hours after transfection). (E) TEAD2 activity in E14/T cells transfected with CA-Yes and/or shRNA against TEAD2 and YAP. All luciferase data are presented as mean \pm s.e.m. percentage of internal control $(n=3) ; * P<0.05$ (ANOVA; Tukey's MC Test).

30 minutes and subjected to electrophoretic mobility shift assays (EMSA) using [ $\left.{ }^{32} \mathrm{P}\right] \mathrm{DNA}$ probes containing the GT-IIC sequence (wt) or a probe containing the same sequence with a single base pair change that eliminates TEAD binding (mut), as previously described (Kaneko and DePamphilis, 1998). As expected, TEAD proteins from ES cell nuclear extracts bind the GT-IIC sequence, but not the mutant sequence (supplementary material Fig. S4A). Moreover, in agreement with the luciferase assays, TEAD DNA binding activity was increased in response to LIF, whereas SU6656 partially inhibited this effect. In addition, a lower TEAD-binding activity was found in 3-week-old embryoid bodies (EBs) compared with that in LIF-stimulated ES cells (supplementary material Fig. S4A,B). In conclusion, these results suggest that LIF activates TEAD2 via Yes and YAP and that this pathway is downregulated in response to differentiation.

\section{Kinase-active Yes associates with YAP and induces its tyrosine phosphorylation}

Interaction between the gp130 subunit of the LIF receptor and the Yes $\mathrm{SH} 2$ domain, but not the $\mathrm{SH} 3$ or unique domains, was induced by LIF in LIF- and serum-starved ES cells as confirmed by mixing cell lysates with GST-SH2-Yes, GST-SH3-Yes, and GST$\mathrm{SH} 3+$ unique-Yes fusion proteins (Fig. 3A). Additionally, coimmunoprecipitation of Yes and YAP was induced by LIF in LIFand serum-starved cells (Fig. 3B). To elucidate whether YAP binds to and is phosphorylated by activated Yes, ES cells were transfected with CA-Yes, immunoprecipitated (IP) with anti-YAP antibody and subjected to western blot analysis for Yes, YAP, 


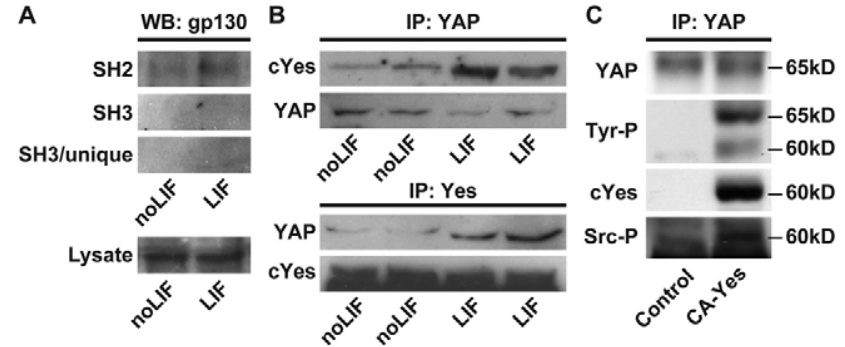

Fig. 3. The SH2 domain of Yes binds gp130 and activated Yes associates with YAP and induces its tyrosine phosphorylation. (A) Affinity absorption of gp130 by GST-tagged fusion proteins expressing the SH2, SH3 and SH3 + unique domains of Yes incubated with cell lysates from AV3 cells that were starved of LIF and serum overnight, and subsequently treated with LIF for 12 hours. Equal input of gp130 in lysates was confirmed by western blotting. (B) E14/T cells starved of LIF and serum overnight and subsequently treated with LIF for 2 hours, immunoprecipitated (IP) with anti-YAP and anti-Yes antibodies and subjected to western blot analysis. (C) E14/T cells transfected with control vector or CA-Yes-expressing vector, immunoprecipitated with anti-YAP antibody and subjected to western blot analysis 48 hours after transfection. Western blots were probed with anti-YAP, anti-PY (Tyr-P), antiYes, or anti-phosphorylated Y416 Src (Src-P, which recognizes all autophosphorylated kinase active SFKs) antibodies.

phosphotyrosine (PY) and PY416-Src (which recognizes all kinaseactive, auto-phosphorylated SFKs) 48 hours after transfection. YAP did co-immunoprecipitate with Yes in cells transfected with CAYes, but did not in the control cells (Fig. 3C). Moreover, despite equal levels of YAP protein in control and CA-Yes-transfected cells, YAP was only tyrosine phosphorylated in cells expressing CA-Yes, suggesting that YAP is a substrate for Yes kinase activity (Fig. 3C).

\section{Yes increases the activity of Oct-3/4 and Nanog promoters in a YAP-dependent manner}

We next addressed the question whether the Yes-YAP-TEAD2 pathway affects promoter activity of the key ES cell regulators Oct3/4 and Nanog. Reporter constructs expressing luciferase driven by the Oct-3/4 (Okumura-Nakanishi et al., 2005) or Nanog promoters (Hattori et al., 2007) were co-expressed with activating or shRNA constructs for Yes, YAP and TEAD2. As expected, high Oct-3/4 and Nanog promoter activities were confirmed in ES cells grown under normal ES cell culture conditions and these were decreased upon LIF withdrawal (data not shown). Moreover, activity of the Oct-3/4 promoter increased as a response to LIF, similarly to what was observed for TEAD2-dependent promoter activities (data not shown). Interestingly, transient transfection of Yes induced a significant increase in Oct-3/4 as well as Nanog promoter activities in ES cells (Fig. 4A,B). Concurrently, expression of shRNA targeting Yes, YAP and TEAD2 resulted in decreases in Oct-3/4 and Nanog promoter activity (Fig. 4A,B) and additionally reduced the LIF-induced activity of the promoters (data not shown). Additionally, shYAP inhibited the Yes-induced activation of both the Oct-3/4 and Nanog promoters, again suggesting that YAP is operating downstream of Yes (Fig. 4C,D).

Because Nanog transcription has previously been shown to be regulated through an interaction between Oct3/4 and SOX2 and their binding to Octamer and SOX elements in the Nanog promoter (Kuroda et al., 2005; Rodda et al., 2005), we next determined whether the effects on Nanog promoter activity was a direct effect
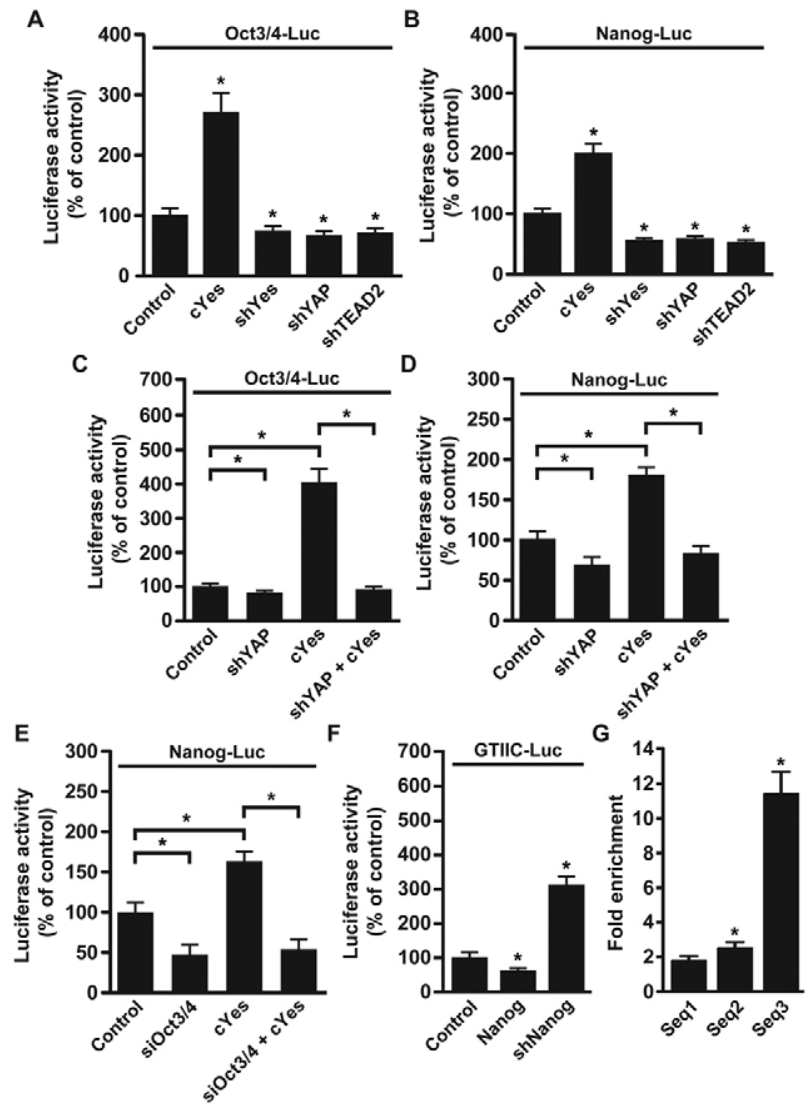

Fig. 4. Yes, YAP and TEAD2 increase Oct-3/4 and Nanog promoter activity. (A) Oct-3/4 and (B) Nanog promoter activity in E14/T cells transfected with Yes and shRNA constructs against Yes, YAP and TEAD2. Oct-3/4 (C) and Nanog (D) promoter activity in E14/T cells transfected with Yes and/or shYAP. (E) Nanog promoter activity in E14/T cells transfected with Yes and/or siOct3/4. (F) TEAD2-dependent transcription (pGTIIC) in E14/T cells transfected with Nanog or shNanog. All Luciferase data are presented as mean \pm s.e.m. percentage of internal control $(n=3) ;{ }^{*} P<0.05$ (ANOVA; Tukey's MC Test). (G) TEAD2 binds the Oct-3/4 promoter. ChIP assays of ES cell chromatin immunoprecipitated with anti-TEAD2 or rabbit IgG (control) and subjected to qPCR using primers for three regions of the mouse Oct-3/4 promoter. Note that Seq. 1 is a control sequence that does not contain any putative TEAD2 binding site. Results are comparative mean \pm s.e.m. $C_{t}$ values $(n=3)$ and results are shown as fold difference in promoter DNA enrichment compared with unspecific binding by IgG. ${ }^{*} P<0.05$ (ANOVA; Tukey's MC Test)

of Yes signaling or an indirect effect caused by the increased expression of Oct3/4. Hence, a Nanog-Luc construct was cotransfected with Yes and small interfering RNA (siRNA) against Oct-3/4 and luciferase activity was measured. Oct-3/4 siRNA almost completely inhibited the Yes-induced activation of the Nanog promoter, implying that Yes signaling activates the Nanog promoter via the induction of Oct3/4 (Fig. 4E). Moreover, to our surprise, overexpression of Nanog significantly decreased the TEAD2-dependent transcriptional activity; whereas knockdown of Nanog with shRNA against Nanog dramatically increased this activity (Fig. 4F), suggesting the presence of a negative-feedback loop regulating YAP and TEAD2 activity in ES cells.

TEAD proteins activate or enhance gene expression by binding to various reporter elements related to the consensus sequence G(A)GA(T/C)ATG in gene promoters or enhancers (Jiang et al., 

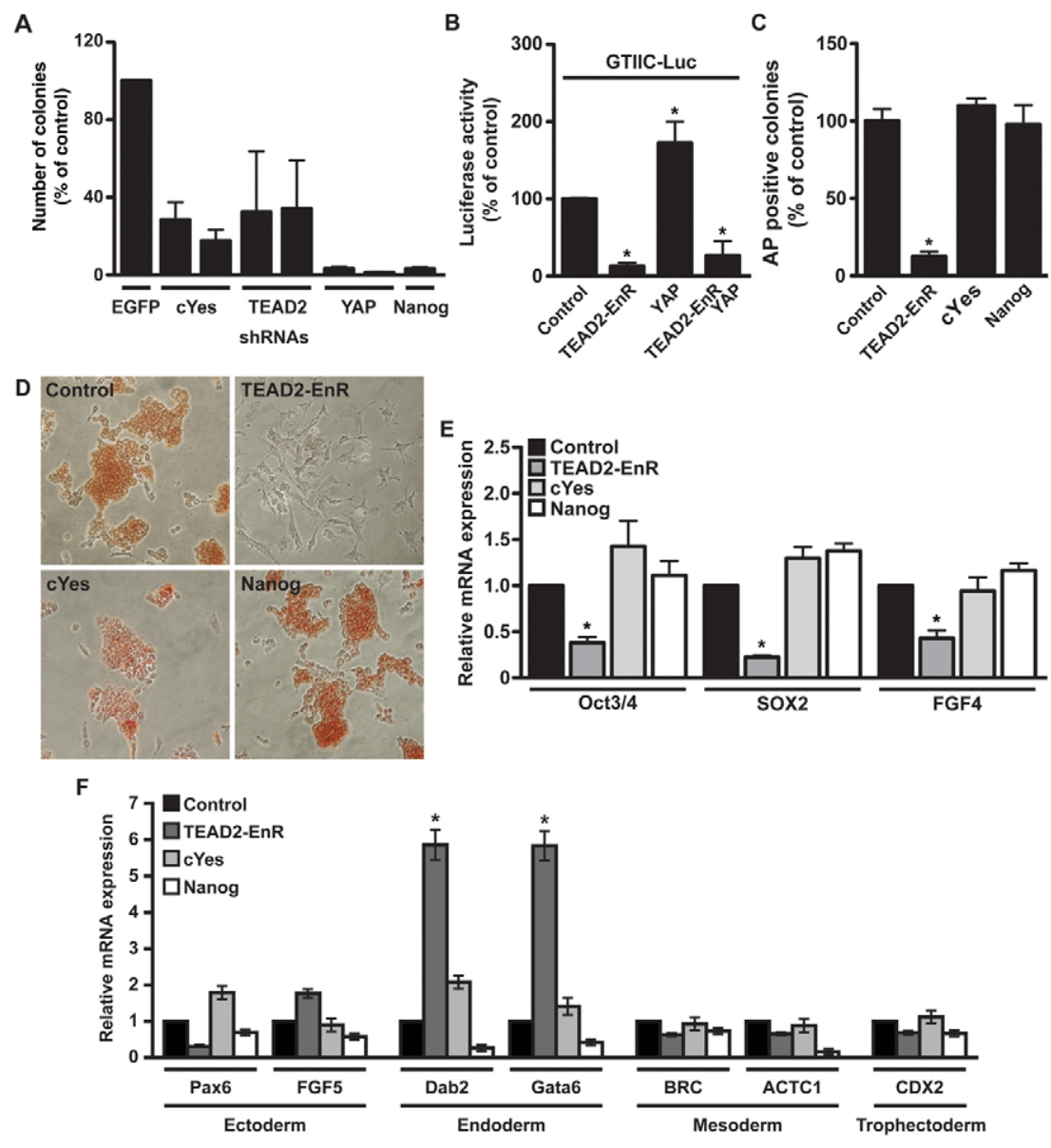

Fig. 5. The Yes-YAP-TEAD2 pathway is necessary for self-renewal of ES cells. (A) RNA interference of Yes, YAP, TEAD2 and Nanog reduces colony-forming ability in ES cells. AV3 cells were transfected with constructs expressing shRNAs against Egfp (control) Yes, Tead2, Yap, or Nanog and a plasmid containing a puromycin-resistance gene. After 2 weeks of puromycin selection $(3 \mathrm{mg} / \mathrm{ml})$, the number of colonies was counted and compared with control shEGFP-transfected cells. Results are mean \pm s.e.m. $(n=3)$. (B) Luciferase assay showing TEAD2dependent activity (pGTIIC) in E14/T cells transfected with YAP and/or TEAD2-EnR. The results are mean \pm s.e.m. $(n=3) ;{ }^{*} P<0.05$ (ANOVA; Tukey's MC Test). (C) Colony formation assays for alkaline phosphatase (AP)-positive E14/T cells. AP-positive colonies were quantified using Image J software. Results are mean \pm s.e.m. $(n=3) ; * P<0.05$ (ANOVA; Tukey's MC Test). (D) Altered morphology and alkaline phosphatase activity staining of E14/T cells 5 days after transfection with TEAD2-EnR compared with cells transfected with empty, Yes and Nanog constructs. (E) qPCR analysis of pluripotency genes in E14/T cells 5 days after transfection with TEAD2-EnR, Yes and Nanog constructs. (F) qPCR analysis of lineage-specific differentiation markers in E14/T cells 5 days after transfection and puromycin selection. For all qPCR analyses, mRNA encoding $\beta$-actin was detected for normalization, controls transfected with empty plasmid were used as a calibrator and results are comparative mean \pm s.e.m. $C_{t}$ values $(n=3) ; * P<0.05$ (ANOVA; Tukey's MC Test).
2000). By searching the $5 \mathrm{~kb}$ upstream genomic region of the Oct$3 / 4$ promoter for these elements, we identified at least two putative TEAD binding sites, at $-1.2 \mathrm{~kb}$ (GGAATG, nt. -1186 to -1181 ; seq. 2) and $-4.7 \mathrm{~kb}$ (CATTCC, nt. -4712 to -4707 ; seq. 3 ; equivalent to GGAATG on the reverse strand) in the Oct-3/4 promoter. Chromatin immunoprecipitation (ChIP) assays were carried out to assess whether TEAD2 associates with any of these regions of the Oct-3/4 promoter. Sequence 1 is a control sequence, overlapping the codon start site and does not contain any TEAD2 binding site. Sequence 2 and sequence 3 overlap the putative -1.2 $\mathrm{kb}$ and $-4.7 \mathrm{~kb}$ binding sites, respectively. In agreement with the luciferase data, the results demonstrated that TEAD2 did indeed bind to the identified response elements in the Oct-3/4 promoter (Fig. 4G).

\section{RNA interference of YAP and TEAD2 reduces formation of ES cell colonies}

We have previously shown that inhibition or knockdown of Yes using chemical inhibitors or shRNAs induces differentiation of ES cells cultured in the presence of LIF and serum (Annerén et al., 2004). To establish whether YAP and TEAD2 are also necessary for ES cell self-renewal, two shRNAs were designed for each gene and expressed in ES cells together with a puromycin selection vector. Nanog shRNA was used as a positive control, whereas shEGFP (enhanced green fluorescent protein) was used as a negative control, as described previously (Annerén et al., 2004). Puromycin-selected colonies were stained for alkaline phosphatase (AP) activity and the number of colonies was assessed as a percentage of the shEGFP control. There were no significant changes in AP staining between cells transfected with shEGFP, and the other shRNA constructs, including shNanog (results not shown). However, cells transfected with shRNA against Yes, TEAD2, YAP and Nanog all generated significantly fewer colonies when compared with control shEGFP-transfected cells (Fig. 5A), suggesting that cells expressing reduced or abolished expression of these genes are unable to form colonies.

\section{Decreased TEAD2 activity induces differentiation}

To study sustained high-level blockade of TEAD2 activity in ES cells, a TEAD2 repressor fusion gene construct was generated (TEAD2-EnR) and supertransfected into E14/T cells using an episomal vector system, as previously described (Chambers et al., 2003). TEAD2-EnR completely repressed both endogenous and YAP-induced TEAD2 activity (Fig. 5B). Interestingly, prolonged expression of TEAD2-EnR in ES cells induced cells to acquire a fibroblast-like morphology, and there was a significant reduction in AP activity compared with that in cells transfected with empty plasmid, Yes or Nanog (Fig. 5C,D). TEAD2-EnR induced a clear decrease in the expression of all tested ES cell pluripotency genes, including Oct3/4, SOX2, FGF4, Nanog and GBX2 (Fig. 5E and results not shown). No phenotypical change could be seen in cells overexpressing TEAD2 without the EnR domain, and qPCR analysis failed to show any significant differences in the pluripotency genes 5 days after transfection (data not shown). We next examined whether cells with impaired TEAD2 activity differentiate in a spontaneous manner, similarly to the differentiation 
pattern seen upon LIF withdrawal, or if they undergo a directed differentiation toward a specific germ layer. The same cDNAs used for the assessment of pluripotency gene expression were analyzed by qPCR for at least two genes specific for each germ layer as well as for trophectoderm differentiation. ES cells induced to differentiate in the absence of LIF for 5 days, showed increased expression of genes specific for all three germ layers (results not shown). Conversely, cells transfected with TEAD2-EnR exhibited a significant increase in expression of endoderm-specific genes (e.g. Dab2 and Gata6), whereas increased expression of other germ-layer-related genes could not be detected (Fig. 5E).

\section{Discussion}

LIF activates four parallel pathways in mouse ES cells: the STAT3, MAPK, PI3K and SFK pathways (Fig. 6). It was recently shown that the STAT3 pathway mainly activates Krueppel-like factor 4 (KLF4), whereas the PI3K pathway stimulates T-box transcription factor (TBX3) (Niwa et al., 2009) and that these in turn activate SOX2 and Nanog, respectively. The MAPK pathway, however, was shown to antagonize the nuclear localization of TBX3 (Burdon et al., 1999; Niwa et al., 2009). In contrast to the other pathways, the SFK pathway has been less well studied and it is not clear how SFKs are linked to the core circuitry of pluripotency-associated transcription factors.

We have previously shown that LIF and serum activate Yes and that inhibition of this pathway disrupts self-renewal of ES cells (Annerén et al., 2004). In the present study, we show that the transcription factor complex YAP-TEAD2 is activated by Yes in ES cells and that TEAD2 in turn regulates expression of the crucial pluripotency genes Oct-3/4 and Nanog. Yes, YAP and TEAD2 undergo dynamic changes in transcriptional and post-transcriptional regulation and activity during mouse ES cell differentiation. Moreover, downregulation of this pathway induces ES cells differentiation.

On the basis of these results, we propose the following model for how Yes, in concert with the above-described LIF-induced pathways, sustains self-renewal in ES cells (Fig. 6). (1) Yes is activated by LIF, through direct binding to activated gp130. (2) Active auto-phosphorylated Yes then transiently associates with and phosphorylates YAP on one or more tyrosine residues, which in turn binds to complexes of TEAD2 and DNA in the nucleus, inducing transcription of genes, including the key pluripotency factor Oct-3/4 . (3) Finally, Oct3/4 activates transcription of Nanog, which in turn, negatively regulates TEAD2 through an unknown mechanism.

We have previously shown that LIF induces Yes kinase activity in mouse ES cells (Annerén et al., 2004). Here, we confirm that the SH2 domain of Yes associates with the LIF receptor subunit gp130. Moreover, we demonstrate that activated Yes associates with YAP and that both proteins in the complex are tyrosinephosphorylated, suggesting that YAP is a direct substrate for Yes. We did not observe any significant changes in the subcellular localization of YAP in response to CA-Yes expression (data not shown), indicating that that Yes-mediated tyrosine phosphorylation of YAP does not affect its nuclear translocation. This contrasts with the well-described serine phosphorylation of YAP by Akt or Lats kinases, which induces binding of YAP to 14-3-3 proteins and cytoplasmic sequestering of YAP (Basu et al., 2003; Zhao et al., 2007). In fact, the role of YAP tyrosine phosphorylation is still unclear. Zaidi and co-workers showed that Src- and Yes-induced tyrosine phosphorylation of YAP is required for interaction of YAP

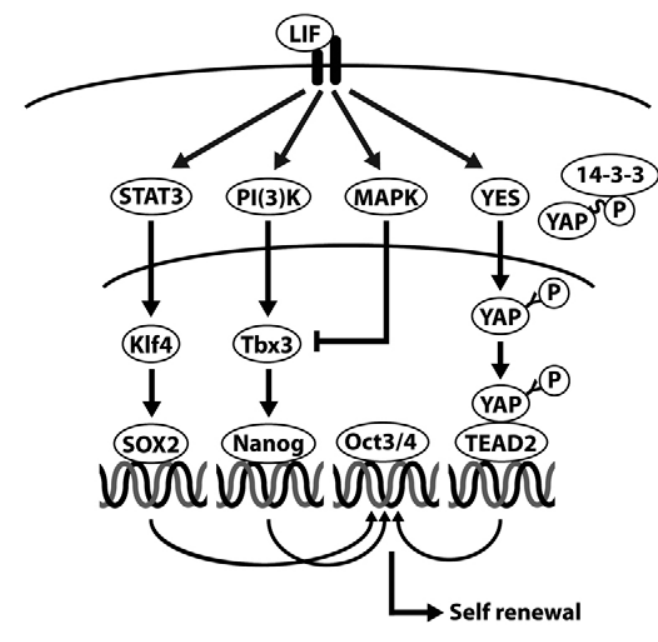

Fig. 6. Model of LIF-regulated self-renewal in mouse ES cells (modified from Niwa et al., 2009). The binding of LIF to the LIFR-gp130 receptor complex leads to recruitment and activation of at least four different downstream signaling pathways: the STAT3 pathway, the MAPK pathway, the PI3K pathway and the Yes pathway. See text for further details.

with the RUNX2 transcription factor in osteoblasts (Zaidi et al., 2004). In line with our observations, they did not observe any clear changes in the nucleocytoplasmic distribution of YAP. Levy and colleagues. demonstrated that Abl-mediated tyrosine phosphorylation of YAP in HEK293 cells stabilizes the YAP protein and increases the affinity of YAP to the p73 transcription factor (Levy et al., 2007). The tyrosine phosphorylation site in YAP was shown to be Y357, which is within the YAP transcriptional activation domain, suggesting that tyrosine phosphorylation directly regulates YAP activity. Interestingly, they also showed that the YAP phosphorylation state influences the p73 specificity of target gene activation, by switching between $\mathrm{p} 73$-mediated pro-apoptotic and growth arrest target genes. Thus, although the exact mechanism of Yes-induced activation of TEAD2 in ES cells remains to be shown, it can be speculated that Yes-induced tyrosine phosphorylation of YAP protects YAP from degradation, increases the affinity of YAP to TEAD2, and/or induces YAP-mediated transcriptional activity.

Additional evidence indicating that Yes directly activates the YAP-TEAD2 pathway includes: (1) transient expression of CAYes or Yes induces TEAD2-dependedent transcription whereas shYes inhibits it; (2) TEAD2 DNA-binding activity is increased in LIF-activated cells, a response that is partially impeded by the SFK inhibitor SU6656; and (3) shYAP inhibits Yes-induced activation of the Oct-3/4 promoter. Our results suggest that the Yes, YAP and TEAD2 pathway is an integral component of the ES cell core regulatory circuitry, a label given to Oct3/4, SOX2 and Nanog because of their co-occupancy of a substantial portion of ES-cell-specific target genes and their involvement in various autoregulatory and feed-forward loops (Boyer et al., 2005; Loh et al., 2006). Interestingly, in a recent study published during revision of this paper, Lian and co-workers presented data strongly supporting our work. For instance, they showed that constitutively active YAP and overexpression of YAP prevented ES cell differentiation. In addition, YAP protein levels were expressed at significantly higher levels in induced pluripotent stem (iPS) cells than in the parental fibroblasts, and addition of YAP to the three 
OSK factors (i.e. Oct3/4, Sox2, Klf4) increased the iPS reprogramming efficiency twofold (Lian et al., 2010). Together, our data mutually corroborate each other and further establish the crucial role of YAP in the self-renewal and differentiation of stem cells.

In the present study, we show that YAP-TEAD2 appears to directly induce Oct3/4 expression by binding to the Oct-3/4 promoter. TEAD2 was found to exhibit strongest binding to the Oct-3/4 promoter region approximately $4.7 \mathrm{~kb}$ upstream of the Oct-3/4 transcriptional start, which, owing to the distant location, is probably enhancer region. This is not surprising because TEAD2 is known to be a strong transcriptional enhancer. Furthermore, transient overexpression and shRNA silencing of Nanog induced a distinct decrease and increase in TEAD2-dependent transcriptional activity, respectively. In addition, previous studies have shown that Yap is one of the top 20 upregulated genes in Oct3/4 tetracycline conditional knockout mouse ES cells (Matoba et al., 2006), and that post-translational modification of Oct3/4 regulates Yes expression by specific binding to the Yes gene promoter (Zhang et al., 2007). Hence, we speculate that the pathways presented in this study form an interconnected autoregulatory loop with Oct $3 / 4$ and Nanog. However, we should emphasize that this needs further investigation.

Interestingly, abnormally high expression levels of kinase-active Yes, YAP or constitutively active mutant TEAD2 (TEAD2-vp16), achieved by supertransfecting E14/T ES cells with episomal vectors, also induce ES cell differentiation (our unpublished observations). These results correlate with results obtained using a similar experimental setup for other key ES cell self-renewal transcription factors such as Tbx3 and Klf4 (Niwa et al., 2009), indicating the tight regulation and dosage effect of these genes.

We additionally show that TEAD2 activity is necessary for ES cell maintenance, because prolonged downregulation of TEAD2 activity induced differentiation. Moreover, this effect was forceful enough to override the LIF- and serum-regulated self-renewal and pluripotency machinery. In conclusion, we propose that Yes, YAP and TEAD2 are part of a tightly controlled signaling pathway downstream of LIF, which regulates the expression of key factors involved in the maintenance and self-renewal of ES cells.

\section{Materials and Methods}

\section{Expression constructs}

A dominant-negative TEAD2 construct was generated by fusing the repression domain of the Drosophila melanogaster homeodomain protein Engrailed to fulllength TEAD2 (TEAD2-EnR) as follows: Full- length mouse Tead2 cDNA was cloned using TOPO ${ }^{\circledR}$ (Invitrogen) immediately upstream of EnR in the SLAX-EnR vector and the TEAD2-EnR fusion gene was then blunt cloned into the XhoI-NotI site of the pPyCAGIP vector. The following expression constructs were also used: pPyCAGIP YAP, pPyCAGIP Nanog, pPyCAGIP TEAD2, pMIK Yes, pMIK Y535F (CA-Yes) (Espanel and Sudol, 2001), pGL3-basic vectors carrying the $2.1 \mathrm{~kb}$ upstream region of the mouse Oct-3/4 gene (the upstream end at nt. -2136 relative to the translational start site) (Okumura-Nakanishi et al., 2005) or the $1 \mathrm{~kb}$ upstream region of mouse Nanog (the upstream end at nt. -983 relative to transcription start site) (Hattori et al., 2007), pCS-luciferase and pCS GT-IIC-luciferase (GTIIC) (Jiang and Eberhardt, 1995) and pCMV $\beta$-gal. The following shRNA sequences are shown in supplementary material Table S1: pSilencer 1.0-U6 shYes, pSuper shYAP, pSilencer 1.0-U6 shTEAD2, pSilencer 1.0-U6 shNanog and pSilencer 1.0-U6 EGFP. Oct3/4 was silenced using siRNA mOct3/4 (sense: GGAUGUGGUUCGAGUAUGGUU) with control ON-TARGETplus non-targeting siRNA to confirm silencing specificity (both from Dharmacon).

\section{Embryonic stem cell culture}

Feeder-dependent 129X1/SvJ-derived AV3 ES cells were cultured on mouse embryonic fibroblasts as previously described (Ramalho-Santos et al., 2002). The E14/T, a mouse embryonic stem cell line constitutively expressing polyoma large-T, was cultivated in $10 \%$ serum (fetal bovine serum and serum replacement in a 50/50 concentration) in the absence of feeder cells, as previously described (Smith, 1991).
For simple differentiation assays, cells were grown in the absence of LIF or in the presence of retinoic acid (RA, $100 \mathrm{nM}$ ) for 72 hours before harvesting.

\section{Electrophoretic mobility shift assay}

Synthetic oligonucleotides (GT-IIC: 5'aagctt-TTCGGGACCCAGGCCTGGAATGTTTCCACC-3' with binding region underlined and GT-IIC Mut: 5'aagcttTCGGGACCCAGGCCTAGAATGTTTCCACC-3') were designed and generated as previously described (Kaneko and DePamphilis, 1998). Purified $\left[\gamma_{-}{ }^{32} \mathrm{P}\right] \mathrm{ATP}-$ labeled GT-IIC and Mut probes were diluted to $20 \mathrm{nM}$ in water and the radioactive signal was checked and normalized with cold oligonucleotide. Cytoplasmic and nuclear extracts were obtained using an NE-PER ${ }^{\mathrm{TM}}$ kit (Pierce) from 3-week-old embryoid bodies, serum starved AV3 ES cells and AV3 cells stimulated with $32 \mathrm{nM}$ LIF for 30 minutes with or without the addition of $4 \mu \mathrm{M}$ SU6656 (Calbiochem; added $30 \mathrm{~min}$ before LIF), according to the manufacturer's instruction. $5 \mu \mathrm{l}$ of nuclear extracts were subjected to SDS-PAGE and stained with Coomassie Blue to ensure equal loading. Equal amounts (approx $5 \mu \mathrm{g}$ ) of nuclear extracts were mixed with reaction buffer (20 mM HEPES pH 7.6, $0.1 \mathrm{mM}$ EGTA, $0.5 \mathrm{mM}$ DTT, $10 \mathrm{mM}$ $\mathrm{NaCl}, 0.1 \mathrm{mg} / \mathrm{ml} \mathrm{dGdC}$ cold competitor, 5\% glycerol and $2 \mu 1\left[\gamma{ }^{32} \mathrm{P}\right]$ ATP-labeled probe), incubated for 15 minutes at room temperature and loaded immediately on a $30 \%$ polyacrylamide (75:1; acrylamide:bis-acrylamide) gel. The gel was subsequently dried and exposed for autoradiography.

\section{Transfection}

Expression constructs (final concentration 3-5 $\mu \mathrm{g}$ DNA/well) were introduced into the mouse ES cells by transfection with Lipofectamine ${ }^{\mathrm{TM}} 2000$ (Invitrogen) according to the manufacturer's recommendations. Cells were incubated at $37^{\circ} \mathrm{C}$ for 4 hours, after which ES cell medium was added (2:1). Cells transfected with episomal vectors (pPyCAGIP) containing a puromycin-resistance gene were selected with $2 \mu \mathrm{g} / \mathrm{ml}$ puromycin (Sigma) for 48 hours to eliminate untransfected cells and then cultured for an additional 72 hours. Colony-forming assays after expression of shRNA and subsequent puromycin selection were done as previously described (Annerén et al., 2004). In short, two different small interfering shRNA sequences for each gene were designed and inserted into pSilencer ${ }^{\mathrm{TM}}$ 1.0-U6 RNA, or pSuper siRNA expression vectors (Ambion). AV3 cells were co-transfected with siRNA constructs and a vector containing a puromycin-resistance gene, cultured for 14 days with puromycin, followed by the counting of colonies and staining for alkaline phosphatase. The shRNA efficiency and auto-phosphorylation of Yes upon ectopic expression of CAYes was assessed 48 hours after transfection without puromycin selection by qPCR and western blotting, respectively. To estimate basal levels of TEAD2 transcriptional activity in ES cells compared with that of differentiated cells, E14/T cells were grown in the absence of LIF with or without the presence of RA (100 nM) for 3 days before transfection with GTIIC-Luc.

\section{Quantitative real-time polymerase chain reaction}

Total RNA was extracted and purified with Qiagen RNeasy Mini kit (Qiagen) according to the manufacturer's instruction. First-strand cDNA was produced according to the manufacturer's protocol with SuperScript ${ }^{\mathrm{TM}} \mathrm{II}$ (Invitrogen) using $1 \mu \mathrm{g}$ RNA and $100 \mathrm{ng}$ random primers (Invitrogen). Quantitative real-time PCR was performed according to the manufacturer's instructions using the Miniopticon ${ }^{\mathrm{TM}}$ Real-Time PCR Detection System (Bio-Rad). The average $C_{t}$ value for each gene was normalized against $\beta$-actin, calibrated against controls transfected with the empty plasmids, and the comparative $C_{t}$ value (fold change) was calculated using $2^{-\Delta \Delta C(t)}$. Transcript level comparison of Tead1, Tead2, Tead3 and Tead4 was based on primer efficiency estimated from five-point dilution curves and used for comparative $C_{t}$ computation according to the PFAFFL method (Pfaffl, 2001).

\section{Alkaline-phosphatase assay}

E14/T cells were transfected, puromycin selected and subsequently grown for 72 hours. Cells were fixed with $4 \%$ paraformaldehyde (PFA) for 1 minute at room temperature and then stained with Vector Red alkaline phosphatase substrate kit (Vector laboratories) according to the manufacturer's instructions. For the quantification of AP-positive colonies, plates were scanned and analyzed with ImageJ software. In parallel with AP analysis, cells were fixed with 4\% PFA for 20 minutes at room temperature and stained with the nuclear stain Hoechst 33342 (Molecular Probes). Cells were mounted with Fluoromount (Sigma) and examined under fluorescent microscope for changes in morphology and nuclear size.

\section{Western blot}

Harvested cells were lysed in lysis buffer (10 mM Tris-HCl, pH 8.0, 1 mM EDTA, $\mathrm{pH} 8.0,150 \mathrm{mM} \mathrm{NaCl}, 0.05 \% \mathrm{NP} 40,0.4 \mathrm{mM}$ PMSF and $1 \mathrm{mM}$ DTT) with the addition of Complete protease inhibitor cocktail (Roche) and $1 \mathrm{mM}$ sodium orthovanadate (Sigma) and subsequently sonicated. Total protein concentration was measured using $\mathrm{BCA}^{\mathrm{TM}}$ Protein Assay Kit (Pierce) to ensure equal loading and subjected to western blot analysis. Membranes were probed with rabbit anti-phosphoSrc family Y416 (1:1000, Cell Signaling), rabbit anti-phospho-YAP S127 (1:1000, Cell Signaling), rabbit anti-YAP (1:1000, Abcam), mouse anti-Yes (1:500, Santa Cruz), mouse anti-phosphotyrosine (1:1000, Millipore), rabbit anti-Yes (1:1000, Cell Signaling), Mouse anti-Stat3 (1:500, Santa Cruz), rabbit anti-phospho-Stat3 T705, rabbit anti-gp130 (1:500, Upstate Biotechnology) and mouse anti-GAPDH (1:2000, 
Santa Cruz), followed by horseradish-peroxidase-conjugated secondary antibodies (1:10,000, Santa Cruz). Immunoreactivity was detected by enhanced chemiluminescence (GE Healthcare).

\section{Immunoprecipitation}

Protein concentration was measured and $1 \mathrm{mg}$ of protein extract was incubated on ice with $2 \mu \mathrm{g}$ rabbit anti-YAP or mouse anti-Yes (Cell Signaling) for 90 minutes, followed by capture with $40 \mu 1$ 1:1 slurry lysis buffer and Immunosorb A (Medicago) for 1 hour at $4^{\circ} \mathrm{C}$. Samples were washed four times with ice-cold lysis buffer, mixed with Laemmli loading buffer, boiled for 5 minutes, and analyzed by western blotting as described above.

\section{Generation of GST fusion proteins}

Gp130 binding experiments were carried out with ES cell lysates and either GSTSH2-Yes, GST-SH3-Yes, and GST-SH3+unique-Yes fusion proteins immobilized on glutathione-Sepharose beads (Summy et al., 2000). Preparation of bacterial lysates containing the encoded fusion proteins, and the absorption to glutathioneSepharose beads were performed as follows: $1 \mathrm{ml}$ sonicated and centrifuged bacterial lysates were mixed with $100 \mu \mathrm{l}$ glutathione-Sepharose 4B (GE Healthcare) for 30 minutes on ice. The beads were washed three times with PBS and 1\% Triton X-100. The beads conjugated to fusion proteins were mixed with ES cell lysates for 1.5 hours on ice, washed with PBS containing Triton, resuspended in Laemmli loading buffer and boiled for 5 minutes. The samples were then subjected to western blotting as described above using anti-gp130 (Millipore). Fusion proteins were quantified by staining with Coomassie Blue.

\section{Luciferase reporter assay}

24 hours after transfection with equal amounts of total plasmid DNA, including empty expression vectors and the pCMV $\beta$-gal reference plasmid containing a bacterial $\beta$-galactosidase gene, cells were harvested and lysed, and extracts were assayed for luciferase and $\beta$-galactosidase activities in a microplate luminometer and photometer reader (Wallac VICTOR 1420 Multilabel Counter: Perkin Elmer). Results are presented as the mean percentage of the control of at least three independent experiments made in triplicate.

\section{Chromatin immunoprecipitation assay}

E14/T DNA and proteins were crosslinked with formaldehyde (final concentration $0.37 \%$ ) for 10 minutes at room temperature, and the reaction was stopped by the addition of $0.125 \mathrm{M}$ glycine for 5 minutes. Chromatin was sheared in shearing buffer (Diagenode) to an average DNA fragment size of $0.5-1 \mathrm{~kb}$ using a Bioruptor sonicator (Diagenode). ChIP assays were performed using One Day ChIP assay kit (Diagenode) according to the manufacturer's protocol. Antibodies used for immunoprecipitation included anti-TEF4 1 polyclonal antibody sc-67115 (Santa Cruz) and IgG (Diagenode) as a negative control. DNA was analyzed by qPCR for various regions of the $O c t-3 / 4$ promoter (primer sequences in supplementary material Table S1) and fold induction over input was calculated using $2^{-\Delta \Delta C(t)}$.

\section{Statistics}

At least three independent experiments were performed and data are presented as mean \pm s.e.m. When applicable one-way analysis of variance (ANOVA) followed by Tukey's multiple comparison post-hoc test was used to evaluate the statistical significance $\left({ }^{*} P<0.05\right)$ of the difference in values using the GraphPad Prism software.

Part of this work was performed in the labs of Douglas Melton, Harvard University and Jonas Frisén, Karolinska Institutet. The following individuals kindly provided reagents: Andrew McMahon, Harvard University (AV3 cells and puro-GFP vector), Ian Chambers and Austin Smith, University of Edinburgh (E14/T cells, pPyCAGIP and pPyCAGIP Nanog vector), Daniel Flynn, West Virginia University School of Medicine (GST-SH2Yes, GST-SH3Yes and GSTSH3+uniqueYes), Norman Eberhardt, Mayo Clinic (the pCS and pGTIIC constructs), Fuyuki Ishikawa, Kyoto University (Oct3/4luciferase constructs), and Kunio Shiota, University of Tokyo (Nanogluciferase construct), Birgitta Tomkinson, Uppsala University (pCMV $\beta$-gal vector), Johan Ericson, Karolinska Institutet (cloning vectors containing EnR and vp16). We would like to thank Fredrik Öberg and Antonia Kalushkova, both at Uppsala University, for valuable technical assistance with the ChIP assay. This work was supported by the Swedish Research Council, the Juvenile Diabetes Research Association, the Swedish Diabetes Association, Jaenssons' Foundations and Faculty of Medicine Uppsala University.

\section{References}

Abram, C. L. and Courtneidge, S. A. (2000). Src family tyrosine kinases and growth factor signaling. Exp. Cell Res. 254, 1-13.

Annerén, C. (2008). Tyrosine kinase signalling in embryonic stem cells. Clin. Sci. (Lond.) $115,43-55$.

Annerén, C., Cowan, C. A. and Melton, D. A. (2004). The Src family of tyrosine kinases is important for embryonic stem cell self-renewal. J. Biol. Chem. 279, 31590-31598.

Basu, S., Totty, N. F., Irwin, M. S., Sudol, M. and Downward, J. (2003). Akt phosphorylates the Yes-associated protein, YAP, to induce interaction with 14-3-3 and attenuation of p73-mediated apoptosis. Mol. Cell 11, 11-23.

Boyer, L. A., Lee, T. I., Cole, M. F., Johnstone, S. E., Levine, S. S., Zucker, J. P., Guenther, M. G., Kumar, R. M., Murray, H. L., Jenner, R. G. et al. (2005). Core transcriptional regulatory circuitry in human embryonic stem cells. Cell 122, 947-956.

Burdon, T., Chambers, I., Stracey, C., Niwa, H. and Smith, A. (1999). Signaling mechanisms regulating self-renewal and differentiation of pluripotent embryonic stem cells. Cells Tissues Organs 165, 131-143.

Burdon, T., Smith, A. and Savatier, P. (2002). Signalling, cell cycle and pluripotency in embryonic stem cells. Trends Cell Biol. 12, 432-438.

Chambers, I., Colby, D., Robertson, M., Nichols, J., Lee, S., Tweedie, S. and Smith, A. (2003). Functional expression cloning of Nanog, a pluripotency sustaining factor in embryonic stem cells. Cell 113, 643-655.

Ernst, M., Gearing, D. P. and Dunn, A. R. (1994). Functional and biochemical association of Hck with the LIF/IL-6 receptor signal transducing subunit gp130 in embryonic stem cells. EMBO J. 13, 1574-1584.

Espanel, X. and Sudol, M. (2001). Yes-associated protein and p53-binding protein-2 interact through their WW and SH3 domains. J. Biol. Chem. 276, 14514-14523.

Hattori, N., Imao, Y., Nishino, K., Ohgane, J., Yagi, S., Tanaka, S. and Shiota, K. (2007). Epigenetic regulation of Nanog gene in embryonic stem and trophoblast stem cells. Genes Cells 12, 387-396.

Ivanova, N. B., Dimos, J. T., Schaniel, C., Hackney, J. A., Moore, K. A. and Lemischka, I. R. (2002). A stem cell molecular signature. Science 298, 601-604.

Jiang, S.-W. and Eberhardt, N. L. (1995). Involvment of a protein distinct from transcription enhancer factor-1 (TEF-1) in mediating human chorionic somatomammotropin gene enhancer function through the GT-IIC enhanson in choriocarcinoma and COS cells. J. Biol. Chem. 270, 13906-13915.

Jiang, S. W., Desai, D., Khan, S. and Eberhardt, N. L. (2000). Cooperative binding of TEF-1 to repeated GGAATG-related consensus elements with restricted spatial separation and orientation. DNA Cell Biol. 19, 507-514

Kaneko, J. K. and DePamphilis, M. L. (1998). Regulation of gene expression at the beginning of mammalian development and the TEAD family of transcription factors. Dev. Genet. 22, 43-66.

Kaneko, J. K., Cullinan, E. B., Latham, K. E. and DePamphilis, M. L. (1997). Transcription factor mTEAD-2 is selectively expressed at the beginning of zygotic gene expression in the mouse. Development 124, 1963-1973.

Kaneko, K. J., Rein, T., Guo, Z. S., Latham, K. and DePamphilis, M. L. (2004). DNA methylation may restrict but does not determine differential gene expression at the Sgy/Tead2 locus during mouse development. Mol. Cell. Biol. 24, 1968-1982.

Kuroda, T., Tada, M., Kubota, H., Kimura, H., Hatano, S. Y., Suemori, H., Nakatsuji, N. and Tada, T. (2005). Octamer and Sox elements are required for transcriptional cis regulation of Nanog gene expression. Mol. Cell. Biol. 25, 2475-2485.

Levy, D., Adamovich, Y., Reuven, N. and Shaul, Y. (2007). The Yes-associated protein 1 stabilizes $\mathrm{p} 73$ by preventing Itch-mediated ubiquitination of $\mathrm{p} 73$. Cell Death Differ. 14, 743-751.

Lian, I., Kim, J., Okazawa, H., Zhao, J., Zhao, B., Yu, J., Chinnaiyan, A., Israel, M. A., Goldstein, L. S., Abujarour, R. et al. (2010). The role of YAP transcription coactivator in regulating stem cell self-renewal and differentiation. Genes Dev. 11, 1106-1118.

Liu, N., Lu, M., Tian, X. and Han, Z. (2007). Molecular mechanisms involved in selfrenewal and pluripotency of embryonic stem cells. J. Cell. Physiol. 211, 279-286.

Loh, Y. H., Wu, Q., Chew, J. L., Vega, V. B., Zhang, W., Chen, X., Bourque, G., George, J., Leong, B., Liu, J. et al. (2006). The Oct4 and Nanog transcription network regulates pluripotency in mouse embryonic stem cells. Nat. Genet. 38, 431-440.

Matoba, R., Niwa, H., Masui, S., Ohtsuka, S., Carter, M. G., Sharov, A. A. and Ko, M. S. (2006). Dissecting Oct3/4-regulated gene networks in embryonic stem cells by expression profiling. PLoS One $\mathbf{1}$, e26.

Meyn, M. A., 3rd and Smithgall, T. E. (2009). Chemical genetics identifies c-Src as an activator of primitive ectoderm formation in murine embryonic stem cells. Sci. Signal. 2 , ra64.

Meyn, M. A., 3rd, Schreiner, S. J., Dumitrescu, T. P., Nau, G. J. and Smithgall, T. E. (2005). SRC family kinase activity is required for murine embryonic stem cell growth and differentiation. Mol. Pharmacol. 68, 1320-1330.

Morin-Kensicki, E. M., Boone, B. N., Howell, M., Stonebraker, J. R., Teed, J., Alb, J. G., Magnuson, T. R., O'Neal, W. and Milgram, S. L. (2006). Defects in yolk sac vasculogenesis, chorioallantoic fusion, and embryonic axis elongation in mice with targeted disruption of Yap65. Mol. Cell. Biol. 26, 77-87.

Nishioka, N., Yamamoto, S., Kiyonari, H., Sato, H., Sawada, A., Ota, M., Nakao, K. and Sasaki, H. (2008). Tead4 is required for specification of trophectoderm in preimplantation mouse embryos. Mech. Dev. 125, 270-283.

Nishioka, N., Inoue, K., Adachi, K., Kiyonari, H., Ota, M., Ralston, A., Yabuta, N., Hirahara, S., Stephenson, R. O., Ogonuki, N. et al. (2009). The Hippo signaling pathway components Lats and Yap pattern Tead4 activity to distinguish mouse trophectoderm from inner cell mass. Dev. Cell 16, 398-410.

Niwa, H., Ogawa, K., Shimosato, D. and Adachi, K. (2009). A parallel circuit of LIF signalling pathways maintains pluripotency of mouse ES cells. Nature 460, 118-122. 
Okumura-Nakanishi, S., Saito, M., Niwa, H. and Ishikawa, F. (2005). Oct-3/4 and Sox 2 regulate Oct-3/4 gene in embryonic stem cells. J. Biol. Chem. 280, 5307-5317.

Pfaffl, M. W. (2001). A new mathematical model for relative quantification in real-time RT-PCR. Nucleic Acids Res. 29, e45.

Ramalho-Santos, M., Yoon, S., Matsuzaki, Y., Mulligan, R. C. and Melton, D. A. (2002). "Stemness": transcriptional profiling of embryonic and adult stem cells. Science 298, 597-600.

Rodda, D. J., Chew, J. L., Lim, L. H., Loh, Y. H., Wang, B., Ng, H. H. and Robson, P. (2005). Transcriptional regulation of nanog by OCT4 and SOX2. J. Biol. Chem. 280, 24731-24737.

Sawada, A., Kiyonari, H., Ukita, K., Nishioka, N., Imuta, Y. and Sasaki, H. (2008). Redundant roles of Tead1 and Tead2 in notochord development and the regulation of cell proliferation and survival. Mol. Cell. Biol. 28, 3177-3189.

Smith, A. (1991). Culture and differentiation of embryonic stem cells. Methods Cell Sci. 13, 89-94.

Sudol, M. (1994). Yes-associated protein (YAP65) is a proline-rich phosphoprotein that binds to the SH3 domain of the Yes proto-oncogene product. Oncogene 9, 2145-2152.

Sudol, M., Chen, H. I., Bougeret, C., Einbond, A. and Bork, P. (1995). Characterization of a novel protein-binding module-the WW domain. FEBS Lett. 369, 67-71.

Summy, J. M., Guappone, A. C., Sudol, M. and Flynn, D. C. (2000). The SH3 and SH2 domains are capable of directing specificity in protein interactions between the nonreceptor tyrosine kinases cSrc and cYes. Oncogene 19, 155-160.
Thomas, S. M. and Brugge, J. S. (1997). Cellular functions regulated by Src family kinases. Annu. Rev. Cell Dev. Biol. 13, 513-609.

Trouillas, M., Saucourt, C., Guillotin, B., Gauthereau, X., Ding, L., Buchholz, F., Doss, M. X., Sachinidis, A., Hescheler, J., Hummel, O. et al. (2009). Three LIFdependent signatures and gene clusters with atypical expression profiles, identified by transcriptome studies in mouse ES cells and early derivatives. BMC Genomics 10, 73.

Vassilev, A., Kaneko, K. J., Shu, H., Zhao, Y. and DePamphilis, M. L. (2001). TEAD/TEF transcription factors utilize the activation domain of YAP65, a Src/Yesassociated protein localized in the cytoplasm. Genes Dev. 15, 1229-1241.

Yagi, R., Kohn, M. J., Karavanova, I., Kaneko, K. J., Vullhorst, D., DePamphilis, M. L. and Buonanno, A. (2007). Transcription factor TEAD4 specifies the trophectoderm lineage at the beginning of mammalian development. Development 134, 3827-3836.

Zaidi, S. K., Sullivan, A. J., Medina, R., Ito, Y., van Wijnen, A. J., Stein, J. L., Lian, J. B. and Stein, G. S. (2004). Tyrosine phosphorylation controls Runx2-mediated subnuclear targeting of YAP to repress transcription. EMBO J. 23, 790-799.

Zhang, Z., Liao, B., Xu, M. and Jin, Y. (2007). Post-translational modification of POU domain transcription factor Oct-4 by SUMO-1. FASEB J. 21, 3042-3051.

Zhao, B., Wei, X., Li, W., Udan, R. S., Yang, Q., Kim, J., Xie, J., Ikenoue, T., Yu, J., Li, L. et al. (2007). Inactivation of YAP oncoprotein by the Hippo pathway is involved in cell contact inhibition and tissue growth control. Genes Dev. 21, 2747-2761.

Zhao, B., Lei, Q. Y. and Guan, K. L. (2008). The Hippo-YAP pathway: new connections between regulation of organ size and cancer. Curr. Opin. Cell Biol. 20, 638-646. 